\section{IN BRIEF}

\section{$\Rightarrow$ BIOMARKERS}

\section{Complement activation precedes classifiable SLE}

A cross-sectional and prospective study found that blood samples from patients with probable systemic lupus erythematosus (pSLE) but not meeting ACR classification criteria $(n=92)$ are more frequently positive for cell-bound complement activation products (CB-CAPs), measured either directly (28\%) or within a multi-analyte assay panel (MAP; $40 \%)$, than for low serum complement levels ( $9 \%$ ). A MAP score $>0.8$ at enrolment predicted transition from pSLE to classifiable SLE within 18 months, and was a better predictor of this transition than other serum biomarkers assessed in the study, including anti-dsDNA antibody positivity and low complement levels. ORIGINAL ARTICLE Ramsey-Goldman, R. et al. Complement activation occurs in patients with probable systemic lupus erythematosus and may predict progression to ACR classified SLE. Arthritis Rheumatol. https://doi.org/10.1002/art.41093 (2019)

\section{CLINICAL TRIALS}

\section{LLDAS is an attainable SLE treatment target}

The findings of an international prospective cohort study validate the lupus low disease activity state (LLDAS) as a treat-to-target endpoint for systemic lupus erythematosus (SLE). In the study, which followed 1,707 adults with SLE for a mean of 2.2 years, attainment of LLDAS at any time point was associated with protection from accrual of irreversible end-organ damage $(0.59,0.45-0.76 ; P<0.0001)$ and disease flares (hazard ratio $0.65,95 \% \mathrm{Cl} 0.56-0.75 ; P<0.0001$ ). Cumulative time in LLDAS and increased durations of sustained LLDAS were also associated with reduced risk of damage accrual.

ORIGINAL ARTICLE Golder, V. et al. Lupus low disease activity state as a treatment endpoint for systemic lupus erythematosus: a prospective validation study. Lancet Rheumatol. https://doi.org/10.1016/\$2665-9913(19)30037-2 (2019)

\section{SYSTEMIC LUPUS ERYTHEMATOSUS}

\section{A new model to study BAFF-independent SLE}

In a new experimental model of systemic lupus erythematosus (SLE), lupus-prone mice with genetic deficiency of B cell activating factor (BAFF) can still develop full-blown disease. The NZM.Baff ${ }^{-1}$ mice express a human BCL2 transgene (Tg) in their $B$ cells, thus preserving $B$ cell survival largely independent of BAFF-triggered signals. In NZM.Baff-1-BCL2 $2^{\text {Tg }}$ mice, immunological, serological and clinical features of SLE developed more rapidly than in NZM wild-type mice. This model could help explain why some patients with SLE fail to respond to anti-BAFF therapy.

ORIGINAL ARTICLE Stohl, W. et al. Development of murine systemic lupus erythematosus in the absence of BAFF. Arthritis Rheumatol. https://doi.org/10.1002/art.41097 (2019)

\section{PSORIATIC ARTHRITIS}

\section{Novel cytokine, IL-41, linked with PsA}

The immunoregulatory cytokine IL-41 (also known as meteorinlike protein) is expressed at high levels in the synovium of patients with psoriatic arthritis ( $\mathrm{PsA})$, compared with patients with osteoarthritis (OA). IL-41 is also present at the enthesis, the primary inflammatory site of PsA, where it is produced primarily by entheseal stromal cells. Expression of IL-41 by entheseal stromal cells can be further induced by stimulation with IL-17A/F and TNF. The functional effects of IL-41 in psoriatic inflammation remain unclear.

ORIGINAL ARTICLE Bridgewood, C. et al. The novel cytokine Metrnl/IL-41 is elevated in psoriatic arthritis synovium and induced at the enthesis. Clin. Immunol. 208, 108253 (2019)

\title{
Is Kawasaki disease a form of IgA vasculitis?
}

Observations in patients and experiments in a well-established mouse model suggest that Kawasaki disease could be a form of IgA vasculitis, in which increased gut permeability leads to leakage of secretory IgA (sIgA) and sIgA-C3 immune complex deposition in cardiovascular lesions. The findings, published in Immunity, also demonstrate a role for IL- $\beta$ in driving intestinal permeability, and suggest that targeting mucosal barrier dysfunction or the IL- $\beta$ pathway could have therapeutic potential in Kawasaki disease and related IgA-mediated diseases.

"The role of gut-related immunity in the induction of inflammation in organ systems distant to the gut has been the subject of intensive investigation," explains corresponding author Moshe Arditi. "Here, we demonstrated that changes in intestinal permeability are closely correlated with the development of disease in distant organ systems."

The investigators observed that mice injected with Lactobacillus casei cell wall extract (LCWE), which develop disease features similar to those of Kawasaki disease, had impaired intestinal barrier function, abnormal expression of tight-junction proteins in the small intestine and gut-vascular barrier dysfunction. Serum concentrations of sIgA and $\operatorname{IgA}$ deposition in the heart and abdominal aortic lesions were increased in LCWE-injected mice as compared with PBS-injected control mice. The study also revealed that the complement protein $\mathrm{C} 3$ and IgA form an immune complex that deposits in the vascular tissues and kidney glomeruli of mice with LCWE-induced Kawasaki disease vasculitis, phenotypically reminiscent of IgA vasculitis and IgA nephropathy.

Notably, sIgA concentrations were also increased in serum from paediatric patients with acute-phase Kawasaki disease (compared with febrile patients without Kawasaki disease) not yet treated with intravenous immunoglobulin (IVIG), and these patients also had signs of intestinal barrier dysfunction and systemic inflammation.

Consistent with the notion that Kawasaki disease could be a form of IgA vasculitis involving a gut-vascular axis, pharmacological blockade of intestinal permeability (with the zonulin inhibitor AT1001 or the myosin light chain inhibitor ML-7) reduced serum sIgA concentrations, IgA tissue deposition and cardiovascular pathology in LCWE-injected mice.

IVIG is known to prevent cardiovascular lesions in Kawasaki disease but the underlying mechanisms are unclear. In LCWE-injected mice, IVIG administration decreased gut permeability and prevented cardiovascular lesions, effects that were accompanied by reduced serum sIgA concentrations and IgA deposition in vascular tissues, suggesting that improved gut barrier function might contribute to the therapeutic effects of IVIG in Kawasaki disease.

"Using genetic and pharmacologic inhibition of IL- $1 \beta$ signalling, we demonstrated that IL-1 $\beta$ lay upstream of disrupted intestinal barrier function, subsequent IgA vasculitis development and cardiac inflammation," adds Arditi.

Together, the findings provide a mechanistic link between the gastrointestinal symptoms observed in patients with Kawasaki disease, such as abdominal pain and diarrhea, and intestinal barrier dysfunction, and suggest potential new treatment targets for Kawasaki disease as well as other diseases such as IgA vasculitis and IgA nephropathy.

Sarah Onuora

ORIGINAL ARTICLE Noval Rivas, M. et al. Intestinal permeability and IgA provoke immune vasculitis linked to cardiovascular inflammation. Immunity 51, 508-521.e6 (2019) 